\title{
ON THE UNCONDITIONALLY GRADIENT STABLE SCHEME FOR THE CAHN-HILLIARD EQUATION AND ITS IMPLEMENTATION WITH FOURIER METHOD*
}

\author{
ANDREW CHRISTLIEB ${ }^{\dagger}$, KEITH PROMISLOW $^{\ddagger}$, AND ZHENGFU XU Z $^{\S}$
}

\begin{abstract}
We implement a nonlinear unconditionally gradient stable scheme by Eyre, within the Fourier method framework for the long-time numerical integration of the Allen-Cahn and CahnHilliard equations, which are gradient flows of the Allen-Cahn energy. We propose a new iterative procedure to solve the nonlinear scheme. When the iterative scheme is applied to the Allen-Cahn equation, we show that the nonlinear iteration is a contractive mapping in the $L^{2}$ norm for large time steps. For the Cahn-Hilliard equation, we establish that the proposed iterative scheme converges with a time step constraint. Further, we numerically demonstrate that the iterative scheme converges for large time steps. The scheme allows for spectral accuracy in space and fast simulation of the dynamics in high dimensions while preserving the discrete form of the energy law. For the general potential well, we present the gradient stable scheme without introducing extra stabilizing terms. Therefore, the numerical error introduced by the operator splitting is reduced to its minimum.
\end{abstract}

Key words. Cahn-Hilliard equation, phase-field model, unconditionally gradient stable scheme, Fourier method, iterative method.

AMS subject classifications. $65 \mathrm{~T} 50,82 \mathrm{C} 26$.

\section{Introduction}

The Cahn-Hilliard equation,

$$
u_{t}=-\Delta\left(\epsilon^{2} \Delta u-u^{3}+u\right)
$$

was originally proposed as a model of phase separation and coarsening in mixtures of binary alloys [3]. Supplemented with appropriate boundary conditions, the CahnHilliard equation on a domain $\Omega \subset \mathbf{R}^{d}$ can be viewed as an $H^{-1}$ gradient flow of the Allen-Cahn energy

$$
\mathcal{E}(u)=\int_{\Omega} \frac{\epsilon^{2}}{2}|\nabla u|^{2}+\frac{1}{4}\left(u^{2}-1\right)^{2} d x .
$$

It has been applied to a wide range of complicated problems involving moving interfaces in materials science and multi-phase fluid dynamics [14, 4, 22, 2]. There is also literature on the theoretical study of the Cahn-Hilliard equation regarding existence, regularity, and boundedness of the solution [8]. Numerical simulations have been carried out with finite element $[7,16,11,12]$, spectral [21], and finite difference methods [13], the discontinuous Galerkin method [18], as well as the local discontinuous Galerkin method [19]. The spatial discretization typically depends upon the boundary conditions, the accuracy desired, and the shape of the domain in consideration. However, in all these situations a major challenge arises in accurately resolving the

${ }^{*}$ Received: February 2, 2012; accepted (in revised form): June 2, 2012. Communicated by John Lowengrub.

${ }^{\dagger}$ Department of Mathematics, Michigan State University, East Lansing, MI 48824, USA (christlieb@math.msu.edu).

${ }^{\ddagger}$ Department of Mathematics, Michigan State University, East Lansing, MI 48824, USA (kpromisl@math.msu.edu).

$\S$ Department of Mathematical Science, Michigan Technological University, Houghton, MI 49931, USA (zhengfux@mtu.edu). 
long-time evolution that is dominated by a slow, diffusively driven coarsening process in which phase-separated domains merge into larger domains. The coarsening dynamics exhibits the late-time asymptotic scaling with characteristic length $L \approx t^{1 / 3}$ [1]. Therefore efficient time marching schemes play essential roles in observing the late-time behavior of the system. An efficient numerical simulation of the end-stages of coarsening demands large time steps. However, to accurately simulate the spinodal phase, small time steps are required. In most cases, an adaptive time stepping strategy is employed to capture the scale separation.

An ideal numerical integration scheme should allow for a time step size only constrained by the accuracy requirement and not by stability. For the coarsening process described by the Cahn-Hilliard equation, this requirement translates to small time steps when there is active phase transition, and large time steps for the periods of slow, diffusive mass transport. Several implicit nonlinear schemes have been designed for the Cahn-Hilliard equation which are unconditionally gradient stable [16], such that the discrete form of the energy decays in time, independent of time step size. However, a second obstacle to time stepping arises from solving the nonlinear equation in an implicit or semi-implicit scheme, namely the nonlinear equation may have multiple solutions. This multiplicity of roots manifests itself as a convergence issue which can typically only be avoided by imposing a time step restriction to ensure a unique solution. For the Cahn-Hilliard model considered in [16] this leads to a restriction of the form $k \leq C \epsilon^{2}$ with $k$ as the time step size.

Our work is motivated by the nonlinear semi-implicit scheme

$$
\frac{u^{n+1}-u^{n}}{k}=-\epsilon^{2} \Delta^{2} u^{n+1}+\Delta\left(u^{n+1}\right)^{3}-\Delta u^{n},
$$

proposed in [9]. The underlying idea is to split the right-hand side into contractive and expansive operators, which correspond to convex and concave components of the energy $\mathcal{E}$. The contractive terms are treated implicitly while the expansive terms are treated explicitly. In [17] it was shown that this formulation leads to an unconditionally gradient stable scheme for a finite difference discretization. We extend this result to a finite element framework. Moreover we present the unconditionally gradient stable scheme for the general potential well without adding stabilizing terms which was suggested in [9]. For a family of semi-implicit parameterized $\theta$-schemes for the Cahn-Hilliard equation, in [17], the authors identified the range of the parameters for which the scheme is gradient stable. It is also pointed out in [17] with numerical evidence that a linearized version of (1.3) suggested in [10] is not gradient stable.

The purpose of suggesting a linearized scheme, from our understanding, is only to avoid solving the nonlinear problem (1.3). It is clear that even if the problem is solved using a finite element or finite difference scheme, the computation of the Newton iteration in a three dimensional simulation will be expensive. When a high order method, such as the Fourier method, is preferred for resolving the interfacial structure [5], the cost for the Newton iteration becomes unacceptable. In order to apply spectrally accurate schemes to the nonlinear equation (1.3), in this paper we propose a direct iterative scheme, which avoids construction of the non-sparse Jacobian and solving a large linear system. The iterative scheme is based on contractive mapping, which we will demonstrate later in Section 2. It is a common practice for a linear or nonlinear discretized equation to be solved with an iteration method. In the case of the Cahn-Hilliard equation, a simple iteration was suggested in [6] to find the solution of a nonlinear stable scheme. However, the iterative scheme only converges for small time steps. 
Here we would like to propose a novel iterative approach directly applied to (1.3) which converges for large time step size. When the iterative scheme is applied to the Allen-Cahn equation, we prove that the nonlinear iteration is a contractive mapping in the $L^{2}$ norm for large time steps. For the Cahn-Hilliard equation, we can establish that the proposed iterative scheme converges with a time step constraint. However, we numerically demonstrate that the iterative scheme converges for large time steps. The convergence is independent of the mesh size and the layer width $\epsilon$, although the convergence rate depends on the time step size. Our approach is different from the work in [15], where extra stabilizing terms were introduced in order to apply the semiimplicit Fourier method (only the leading linear term being implicit). In this sense, our approach is more accurate in handling time discretization since numerical error due to operator splitting is reduced to its minimum. Even though the iterative scheme is implemented within the Fourier method framework in our numerical simulation, it can be easily used within other frameworks such as finite element or finite difference. Since we are solving the equation (1.3) directly, the discrete energy law, which the total energy computed numerically always decreases, is preserved.

The rest of the paper is organized as follows. In Section 2, we will review the semi-implicit nonlinear scheme and its basic properties. We give a simple proof that the scheme is unconditionally gradient stable and the nonlinear problem has a unique solution in the classical Sobolev space. We propose an iterative method which can be used to solve (1.3) conveniently. We prove (under a small time step assumption) that the iteration is a contractive mapping under $L^{2}$ norm. However, the purpose of our iterative scheme is to simulate the problem with large time steps. To illustrate the idea, we show that the iteration is a contractive mapping when applied to the AllenCahn equation, with large time steps independent of $\epsilon$ and mesh size. We present the gradient stable scheme for the general potential well. In Section 3, we provide numerical evidence that indeed the nonlinear iteration converges for the Cahn-Hilliard equations even for very large time steps. Numerically, we extend our discussion to the impact of numerical error on the simulated dynamics, when large time steps are used.

\section{Splitting and stability analysis}

In this section we consider the Allen-Cahn and Cahn-Hilliard equations, and the semi-implicit time integration proposed in [9] to solve them. The important feature of the Allen-Cahn and Cahn-Hilliard equations is that they are viewed as the gradient flow of the Allen-Cahn energy functional (1.2). The Allen-Cahn and Cahn-Hilliard equations read as

$$
\frac{d u}{d t}=\epsilon^{2} \Delta u-u^{3}+u
$$

and

$$
\frac{d u}{d t}=-\epsilon^{2} \Delta^{2} u+\Delta\left(u^{3}-u\right)
$$

respectively, with proper boundary and initial conditions. The total energy of the system defined by (1.2) experiences $L^{2}$ and $H^{-1}$ energy decay when its dynamics are associated with (2.1) and (2.2). It is important to design efficient and accurate numerical schemes which preserve the corresponding energy law. From now on, for simplicity, we will assume periodic boundary conditions on a rectangular domain, without loss of generality of our approach. In this paper, we focus on the discussion 
of the Ginzburg-Landau double-well potential $\frac{1}{4}\left(u^{2}-1\right)^{2}$. We will use this potential well as an example to illustrate our approach. We see no difficulty in generalizing our approach to the other forms of the potential well. In the following discussion, we will focus on the Cahn-Hilliard equation and mention the Allen-Cahn equation when necessary. We now introduce notations which will be used throughout the paper. We will use $\langle\cdot, \cdot\rangle$ for the standard $L^{2}$ inner product and $\|\cdot\|_{0}$ for the $L^{2}$ norm. We denote the $L^{\infty}$ norm by $\|\cdot\|_{\infty} . H^{2}(\Omega)$ and $H^{1}(\Omega)$ are the standard Sobolev spaces. Let $\bigcup_{k_{h}}$ be a partition of the domain $\Omega$ and $H_{h}^{1}(\Omega)$ be the corresponding continuous finite element subspace of $H^{1}(\Omega)$ given the partition $\bigcup_{k_{h}}$. Here $r$ is the order of the polynomial on the segment $k_{h}$.

2.1. Stability and solvability. A scheme applied to (2.2) is unconditionally gradient stable if the following discrete form of the energy law is preserved for any time step $k$ :

$$
\mathcal{E}\left(u^{n+1}\right) \leq \mathcal{E}\left(u^{n}\right) .
$$

The scheme of interest is the nonlinear semi-implicit scheme for the Cahn-Hilliard equation proposed in [9]:

$$
\frac{u^{n+1}-u^{n}}{k}=-\epsilon^{2} \Delta^{2} u^{n+1}+\Delta\left(u^{n+1}\right)^{3}-\Delta u^{n},
$$

where $k$ is the time step. We look for the solution $u^{n+1}$ in the Sobolev space $H^{2}(\Omega)$ such that

$$
\left\langle\frac{u^{n+1}-u^{n}}{k}, \phi\right\rangle=-\epsilon^{2}\left\langle\Delta u^{n+1}, \Delta \phi\right\rangle-\left\langle\nabla\left(u^{n+1}\right)^{3}-\nabla u^{n}, \nabla \phi\right\rangle
$$

holds for any $\phi \in H^{2}(\Omega)$. We would like to give a simple proof that (2.3) is unconditionally gradient stable without considering the spatial discretization (for the highly dissipative equation, spatial discretization has less impact on the time step size restriction), namely $\mathcal{E}\left(u^{n+1}\right) \leq \mathcal{E}\left(u^{n}\right)$ for any $n$ and time step $k$. To prove stability, we introduce the operator $\Delta^{-1}$, which is defined such that

$$
\Delta^{-1} u=f, \quad \int u d x=0
$$

is equivalent to

$$
\langle\Delta f, v\rangle=\langle u, v\rangle
$$

for any $v \in L^{2}(\Omega)$. Notice the mapping is not unique in $H^{2}$. Letting $\phi=\Delta^{-1}\left(u^{n+1}-\right.$ $\left.u^{n}\right)$, which is well defined since $\int u^{n+1}-u^{n} d x=0$ due to the fact that (2.4) is in conservative form with periodic boundary conditions, we have

$$
\begin{aligned}
-\frac{1}{k}\left\|\nabla \cdot \Delta^{-1}\left(u^{n+1}-u^{n}\right)\right\|_{0}^{2}=-\epsilon^{2} & \left\langle\Delta u^{n+1}, u^{n+1}-u^{n}\right\rangle \\
& +\left\langle\left(u^{n+1}\right)^{3}-u^{n}, u^{n+1}-u^{n}\right\rangle .
\end{aligned}
$$

Therefore

$$
-\epsilon^{2}\left\langle\Delta u^{n+1}, u^{n+1}-u^{n}\right\rangle+\left\langle\left(u^{n+1}\right)^{3}-u^{n}, u^{n+1}-u^{n}\right\rangle \leq 0 .
$$


Integrating by parts, we have

$$
\epsilon^{2}\left\langle\nabla u^{n+1}, \nabla\left(u^{n+1}-u^{n}\right)\right\rangle+\left\langle\left(u^{n+1}\right)^{3}-u^{n}, u^{n+1}-u^{n}\right\rangle \leq 0 .
$$

By using $a^{2}-a b=\frac{a^{2}}{2}-\frac{b^{2}}{2}+\frac{(a-b)^{2}}{2}$, it follows after a simple calculation that

$$
\begin{aligned}
\epsilon^{2}\left(\frac{1}{2}\left\|\nabla u^{n+1}\right\|_{0}^{2}-\frac{1}{2}\left\|\nabla u^{n}\right\|_{0}^{2}\right)+\left\langle\left(u^{n+1}\right)^{3} \cdot\left(u^{n+1}-u^{n}\right), 1\right\rangle & \\
& +\frac{1}{2}\left\|u^{n}\right\|_{0}^{2}-\frac{1}{2}\left\|u^{n+1}\right\|_{0}^{2} \leq 0 .
\end{aligned}
$$

Using the same identity, we observe that the term $\left(u^{n+1}\right)^{3} \cdot\left(u^{n+1}-u^{n}\right)$ is larger than

$$
\left(u^{n+1}\right)^{2}\left(\frac{1}{2}\left(u^{n+1}\right)^{2}-\frac{1}{2}\left(u^{n}\right)^{2}\right) \geq \frac{1}{4}\left(u^{n+1}\right)^{4}-\frac{1}{4}\left(u^{n}\right)^{4} .
$$

Combining the last two inequalities, we conclude that

$$
\mathcal{E}\left(u^{n+1}\right)-\mathcal{E}\left(u^{n}\right) \leq 0,
$$

and therefore the nonlinear semi-implicit scheme is unconditionally gradient stable. One can prove the uniqueness of the solution to (2.3) in a similar way, as follows.

Lemma 2.1. The time discretized problem (2.4) has at most one solution in $H^{2}(\Omega)$.

Proof. Assuming $u^{n+1}=w, v$ are both solutions, by subtraction

$$
\left\langle\frac{w-v}{k}, \phi\right\rangle=-\epsilon^{2}\langle\Delta(w-v), \Delta \phi\rangle-\left\langle\nabla\left(w^{3}-v^{3}\right), \nabla \phi\right\rangle
$$

holds for any $\phi \in H^{2}(\Omega)$. Let $\phi=\Delta^{-1}(w-v)$. Then the above expression reads as follows:

$$
-\frac{1}{k}\left\|\nabla \Delta^{-1}(w-v)\right\|_{0}^{2}-\epsilon^{2}\|\nabla(w-v)\|_{0}^{2}=\left\langle w^{3}-v^{3}, w-v\right\rangle \geq 0,
$$

thus $w=v$.

This proves the uniqueness of the solution to (2.4).

To summarize, when solved by the finite element method, (2.4) produces a unique energy descent solution for any time step size. The Newton iteration applied to this nonlinear equation should also converge to the correct solution. However the spectral Fourier method has the advantage of not only easy implementation, but also usually providing more accurate results for interface problems using fewer grid points than the finite element and finite difference methods. This advantage is discussed in [16]. The nonlinear terms in (2.4) are the only obstacle to applying the Fourier method. Therefore, we propose an iterative method to solve (2.3).

2.2. Nonlinear iteration: contractive mapping. Generally, to solve the nonlinear problem (2.3), we define

$$
G\left(u^{n+1}\right)=u^{n+1}-u^{n}+k \epsilon^{2} \Delta^{2} u^{n+1}-k \Delta\left(u^{n+1}\right)^{3}+k \Delta u^{n} .
$$

To solve $G\left(u^{n+1}\right)=0$, the Newton iteration requires the Frechet derivative defined as

$$
G^{\prime}\left(u^{n+1}\right) v=v+k \epsilon^{2} \Delta^{2} v-k \Delta 3\left(u^{n+1}\right)^{2} v
$$


with $v$ as the perturbation. The Newton iteration will then be

$$
G^{\prime}\left(u^{r}\right)\left(u^{r+1}-u^{r}\right)=-G\left(u^{r}\right)=-\left(u^{r}-u^{n}+k \epsilon^{2} \Delta^{2} u^{r}-k \Delta\left(u^{r}\right)^{3}+k \Delta u^{n}\right) .
$$

Notice that $G^{\prime}\left(u^{r}\right)$ depends on $u^{r}$, the space variables, making the Fourier method impractical considering the heavy cost of convolution in space. To have a fast simulation, instead of using $G^{\prime}\left(u^{r}\right)$, we seek its approximation

$$
\tilde{G}^{\prime}\left(u^{r}\right) v=v+k \epsilon^{2} \Delta^{2} v-k C \Delta v,
$$

with properly chosen $C>0$. The principle employed to choose the constant $C$ will be discussed later. Therefore the $\tilde{G}^{\prime}\left(u^{r}\right)$ does not depend on $u^{r}$ at all. This makes the fast Fourier method applicable. The Newton iteration is changed to the following fixed point iteration:

$$
\left(1+k \epsilon^{2} \Delta^{2}-k C \Delta\right) u^{r+1}=k \Delta\left(u^{r}\right)^{3}-k C \Delta u^{r}+u^{n}-k \Delta u^{n} .
$$

We shall prove that (2.11) is contractive mapping with an improved time step size $k$. The proof is in the finite element framework, even though our implementation is in the Fourier method framework. First we redefine the problem (2.11) as

$$
\left\{\begin{array}{l}
U-k C \Delta U-k \Delta P=v, \\
P+\epsilon^{2} \Delta U=u^{3}-C u-v,
\end{array}\right.
$$

with $v=u^{n}$ and $\|v\|_{0}=\alpha$. For the partition $\bigcup_{k_{h}}$, in the finite element solution space $H_{h}^{1}(\Omega)$ we look for a pair $U, P$ such that

$$
\left\{\begin{array}{l}
\langle U, \phi\rangle+k C\langle\nabla U, \nabla \phi\rangle+k\langle\nabla P, \nabla \phi\rangle=\langle v, \phi\rangle, \\
\langle P, \psi\rangle-\epsilon^{2}\langle\nabla U, \nabla \psi\rangle=\left\langle u^{3}-C u-v, \psi\right\rangle
\end{array}\right.
$$

holds for any $\phi, \psi \in H_{h}^{1}(\Omega)$. We define a mapping $\Pi_{v}: H_{h}^{1}(\Omega) \rightarrow H_{h}^{1}(\Omega), v \in H_{h}^{1}(\Omega)$, $U=\Pi_{v}(u)$. We would like to show that

Lemma 2.2. For given $\|v\|_{0}=\alpha$, there exists a constant $\beta>0$ and $C>0$ such that $\Pi_{v}$ maps from the ball $S=\left\{u \in H_{h}^{1}(\Omega):\|u\|_{0} \leq 2 \alpha\right\}$ into itself if $k \leq \beta \epsilon^{2} h_{\delta} . h_{\delta}$ is the mesh size related parameter.

Proof. Let $\phi=\epsilon^{2} U$ and $\psi=k P$ in (2.13). We have the following:

$$
\left\{\begin{array}{l}
\epsilon^{2}\|U\|_{0}^{2}+k C \epsilon^{2}\|\nabla U\|_{0}^{2}+k \epsilon^{2}\langle\nabla P, \nabla U\rangle=\epsilon^{2}\langle v, U\rangle, \\
k\|P\| \|_{0}^{2}-k \epsilon^{2}\langle\nabla U, \nabla P\rangle=k\left\langle u^{3}-C u-v, P\right\rangle .
\end{array}\right.
$$

Adding the two equations in (2.14), we have

$$
\frac{\epsilon^{2}}{2}\|U\|_{0}^{2}+k C \epsilon^{2}\|\nabla U\|_{0}^{2}+k\|P\|_{0}^{2} \leq \frac{\epsilon^{2}}{2}\|v\|_{0}^{2}+k\left\langle u^{3}-C u-v, P\right\rangle,
$$

or

$$
\|U\|_{0}^{2}+2 k C\|\nabla U\|_{0}^{2}+\frac{2 k}{\epsilon^{2}}\|P\|_{0}^{2} \leq\|v\|_{0}^{2}+\frac{2 k}{\epsilon^{2}}\left\langle u^{3}-C u-v, P\right\rangle .
$$

Using Young's inequality, it follows that

$$
\|U\|_{0}^{2}+2 k C\|\nabla U\|_{0}^{2} \leq\|v\|_{0}^{2}+\frac{k}{\epsilon^{2}}\left\|u^{3}-C u-v\right\|_{0}^{2} .
$$


By inverse estimates in the finite element space, we have that there is a $\tilde{C}>0$, independent of $h_{\delta}$ and $\alpha$ such that $\|u\|_{\infty} \leq \tilde{C} \alpha h_{\delta}^{-1 / 2}$ for $\|u\|_{0} \leq 2 \alpha$, where $h_{\delta}$ only depends on the mesh size. $\tilde{C}$ only depends on the degree of the underlying polynomial. Here we can choose $C=\left(\tilde{C} \alpha h_{\delta}^{-1 / 2}\right)^{2}$, so that

$$
\begin{aligned}
\|U\|_{0}^{2}+2 k C\|\nabla U\|_{0}^{2} & \leq\|v\|_{0}^{2}+\frac{2 k C^{2}}{\epsilon^{2}}\|u\|_{0}^{2}+\frac{2 k}{\epsilon^{2}}\|v\|_{0}^{2} \\
& \leq \alpha^{2}+\frac{8 k C^{2} \alpha^{2}}{\epsilon^{2}}+\frac{2 k \alpha^{2}}{\epsilon^{2}} .
\end{aligned}
$$

It suffices to require that $8 k C^{2}+2 k \leq 3 \epsilon^{2}$, or equivalently $k \leq \frac{3 \epsilon^{2}}{8 C^{2}+2} \leq \beta \epsilon^{2} h_{\delta}$ for some $\beta>0$.

Similarly we can show

Lemma 2.3. $\Pi_{v}$ is a contractive mapping if $k \leq \beta \epsilon^{2} h_{\delta}$ for some $\beta>0$.

The proof is very similar to the above, hence we will not repeat it. However, we would like to add comments here on this estimate:

1. The estimate is based on the "non-optimal" inverse maximal value estimate on a bounded $L^{2}$ subset of the finite element space $H_{h}^{1}(\Omega)$. Computationally, a relatively larger $k$ might be allowed.

2. The estimate is restrictive considering the $\epsilon^{2} h_{\delta}$ constraint, even though the term $2 k C\|\nabla U\|_{0}^{2}$ on the left hand side gives a larger convergence radius. Unfortunately the result is the best we have obtained to date and it is not much better than the conclusion in [6].

3. The idea of adding the term $-k C \Delta U$ is to linearize a contractive operator.

4. The numerical results show that there is no obvious restriction by $\epsilon$ and the mesh size $h_{\delta}$.

5. The iterative approach can be applied to most of the $\theta$ schemes discussed in [17] and for the general double-well potential with the stabilized splitting.

If we apply this technique to the Allen-Cahn equation, we can gain insight into how this technique works. Consider the dynamics described by the Allen-Cahn equation (2.1). Its corresponding nonlinear semi-implicit scheme is

$$
\frac{u^{n+1}-u^{n}}{k}=\epsilon^{2} \Delta u^{n+1}-\left(u^{n+1}\right)^{3}+u^{n} .
$$

Now, we apply the technique in (2.11) to the semi-discretized equation (2.19):

$$
\left(1-k \epsilon^{2} \Delta+k C\right) u^{r+1}=-k\left(u^{r}\right)^{3}+k C u^{r}+u^{n}+k u^{n} .
$$

Again, let $U=u^{r+1}, u=u^{r}, v=u^{n}$. For convenience, we consider this problem in the finite element formulation

$$
\langle U, \phi\rangle+k \epsilon^{2}\langle\nabla U, \nabla \phi\rangle+k C\langle U, \phi\rangle=k\left\langle\left(C-u^{2}\right) u, \phi\right\rangle+(1+k)\langle v, \phi\rangle .
$$

We shall focus on the contractive property only. Assuming $\|v\|_{0}=\alpha$ and $S=\{u \in$ $\left.H_{h}^{1}(\Omega):\|u\|_{0} \leq 2 \alpha\right\}$, by $(2.21)$ we define a mapping $U=\Pi_{v}(u)$. We would like to show the following result. 
Lemma 2.4. $\Pi_{v}$ maps $S$ onto itself with $C=\max \left\{3|| u \|_{\infty}^{2} \mid u \in S\right\}$ for $k \leq 1$, with $\|u\|_{\infty} \leq \tilde{C} \alpha h_{\delta}^{-1 / 2}$ for $\|u\|_{0} \leq 2 \alpha$.

Proof. Let $\phi=U$. It follows that

$$
\langle U-(1+k) v, U\rangle+k \epsilon^{2}\|\nabla U\|_{0}^{2}+k C\left\langle U-\frac{C-u^{2}}{C} u, U\right\rangle=0 .
$$

Therefore,

$$
\frac{1}{2}\|U\|_{0}^{2}-\frac{(1+k)^{2}}{2}\|v\|_{0}^{2}+k \epsilon^{2}\|\nabla U\|_{0}^{2}+\frac{k C}{2}\|U\|_{0}^{2}-\frac{k C}{2}\|u\|_{0}^{2} \leq 0
$$

since $0 \leq \frac{C-u^{2}}{C} \leq 1$. To prove the lemma it suffices to require $\frac{4 k C \alpha^{2}+(k+1)^{2} \alpha^{2}}{1+k C} \leq 4 \alpha^{2}$ or $(k+1)^{2} \leq 4$, i.e $k \leq 1$. Thus $\Pi_{v}$ maps $S$ into itself.

Next we show it is indeed contractive.

Lemma 2.5. $\Pi_{v}$ is a contractive mapping in $S$ when $k \leq 1$.

Proof. Let $U_{u}, U_{w}$ be the solutions corresponding to $u, w \in S$. By subtraction and the Mean Value Theorem,

$$
\begin{aligned}
& \left\langle U_{u}-U_{w}, \phi\right\rangle+k \epsilon^{2}\left\langle\nabla\left(U_{u}-U_{w}\right), \nabla \phi\right\rangle+k C\left\langle U_{u}-U_{w}, \phi\right\rangle \\
= & k\left\langle\left(C-u^{2}\right) u-\left(C-w^{2}\right) w, \phi\right\rangle \\
= & k\left\langle\left(C-3 \tilde{u}^{2}\right)(u-w), \phi\right\rangle .
\end{aligned}
$$

Let $\phi=U_{u}-U_{w}$. Noticing that $\frac{C-3 \tilde{u}^{2}}{C} \leq 1$, a simple calculation gives

$$
\left\|U_{u}-U_{w}\right\|_{0}^{2}+\frac{k C}{2}\left\|U_{u}-U_{w}\right\|_{0}^{2} \leq \frac{k C}{2}\|u-w\|_{0}^{2},
$$

which simply implies that

$$
\left\|U_{u}-U_{w}\right\|_{0} \leq\left(\frac{k C / 2}{1+k C / 2}\right)^{1 / 2}\|u-w\|_{0},
$$

and thus the mapping is contractive.

From $(2.26)$ we can find the contraction rate $\left(\frac{k C / 2}{1+k C / 2}\right)^{1 / 2}$, which depends on the point-wise maximal value and time step size. However, it is not straightforward to establish such an estimate for the Cahn-Hilliard equation, even though numerically we observe a similar convergence rate in that case. There should be no difficulty in generalizing this iterative approach to the class of $\theta$ schemes [17] for the CahnHilliard equation. In the next section, for general potential wells $W(u)$, we will discuss the gradient splitting, without introducing stabilizing terms, and the corresponding iterative scheme.

2.3. Gradient stable scheme for general Cahn-Hilliard equation. For the more general potential-well, the Cahn-Hilliard equation reads as

$$
\frac{d u}{d t}=-\epsilon^{2} \Delta^{2} u+\gamma_{2} \Delta u^{3}+\gamma_{1} \Delta u^{2}+\gamma_{0} \Delta u
$$

where $\gamma_{2}>0$ to ensure existence of a minimum energy. The parameters are normally decided by the background material. Without loss of generality, we assume $\gamma_{i}>0, i=$ 
$0,1,2$. To implement the contractive and expansive splitting without introducing extra stabilizing terms, we rewrite $(2.27)$ as

$$
\frac{d u}{d t}=-\epsilon^{2} \Delta^{2} u+\gamma_{2} \Delta u^{3}+\gamma_{1} \Delta\left(H^{+}(u)+H^{-}(u)\right)^{2}+\gamma_{0} \Delta u
$$

with

$$
H^{+}(u)= \begin{cases}u, & u>0 \\ 0, & u \leq 0\end{cases}
$$

and

$$
H^{-}(u)= \begin{cases}0, & u \geq 0 \\ u, & u<0\end{cases}
$$

After splitting the gradients, the equation (2.28) can be rewritten as

$$
\frac{d u}{d t}=-\epsilon^{2} \Delta^{2} u+\gamma_{2} \Delta u^{3}+\gamma_{1} \Delta H^{+}(u)^{2}+\gamma_{1} \Delta H^{-}(u)^{2}+\gamma_{0} \Delta u
$$

Noticing that $H^{+}(u)^{2}$ and $H^{-}(u)^{2}$ are the gradients of the twice differentiable convex and concave energy forms $\frac{H^{+}(u)^{3}}{3}$ and $\frac{H^{-}(u)^{3}}{3}$ respectively, the unconditionally gradient stable semi-implicit scheme can be designed as

$$
\begin{aligned}
& \frac{u^{n+1}-u^{n}}{k}=-\epsilon^{2} \Delta^{2} u^{n+1}+\gamma_{2} \Delta\left(u^{n+1}\right)^{3} \\
& +\gamma_{1} \Delta H^{+}\left(u^{n+1}\right)^{2}+\gamma_{1} \Delta H^{-}\left(u^{n}\right)^{2}+\gamma_{0} \Delta u^{n+1}
\end{aligned}
$$

For a different group of constants $\gamma_{i}, i=0,1,2$, the scheme can be written out correspondently. The proof that the scheme (2.32) is unconditionally gradient stable is same as the symmetric potential-well case after observing that the $\left(H^{ \pm}(u)\right)^{2}$ falls into the category of being a contractive or expansive gradient, respectively. We will not repeat it here. To solve the nonlinear equation with the Fourier method, there is no essential difficulty to extend the iterative approach (2.11) to the nonlinear scheme (2.32).

2.4. Accuracy and other issues. Usually the semi-implicit scheme based on convex-concave splitting is less accurate than the forward and backward Euler schemes. The semi-implicit nonlinear scheme described in this paper produces at most a first order accurate solution. The splitting error of the semi-implicit method can impact the accuracies of the simulated dynamics. However, first, since the scheme preserves the discrete energy law, the impact of the splitting error is not detrimental; second, for a long time evolution system, when the simulation is close to steady state, the accuracy of the time discretization is no longer as important. The space discretization accuracy determines the resolution of the interface. In our case the Fourier method produces the fine resolution of interfacial structures. The estimate of the contraction rate indicates that the convergence is slower than Newton iterations. The trade off is that a high resolution Fourier method can be easily applied. Within the Fourier method framework, compared with the Newton iteration, the iterative method does not require solving a large linear system with non-sparse matrix. This is a very appealing advantage when solving three dimensional problems. In one and two 
dimensions, the simulation can be run on a PC to obtain results at almost every desired time stage. For the three dimensional simulation, since most of the computational cost is in the FFT operation, parallel FFT will be the ideal choice to speed up the simulation.

\section{Numerical result}

In the simulation, we use the discrete Fourier method with adaptive time steps. The adaptive time stepping strategy is based on the computation of the right hand side of equation (2.2). After each time-marching step, we compute the right hand side of (2.2), denoted by $R\left(u^{n}\right)$. We measure the maximal value of $R\left(u^{n}\right)$, which is an indicator of how far the current state is away from steady state (the minimal energy morphology). The time step size is determined by $k=\max \left(\frac{\tau}{\left\|R\left(u^{n}\right)\right\|_{\infty}}, \gamma\right)$, with the freedom of choice of $\tau>0$. The constant $\gamma$, which is $10^{-6}$ in our simulation, is set to be the minimal time step to avoid excessively small time steps.

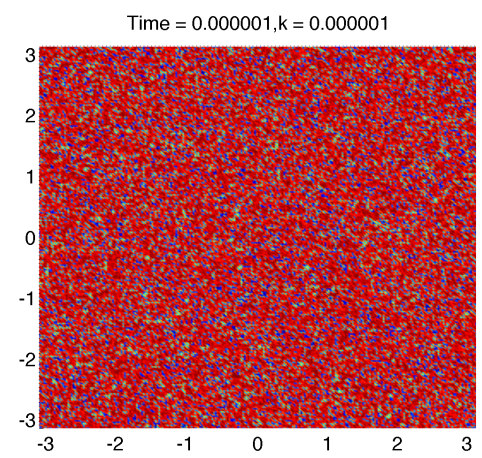

(a)

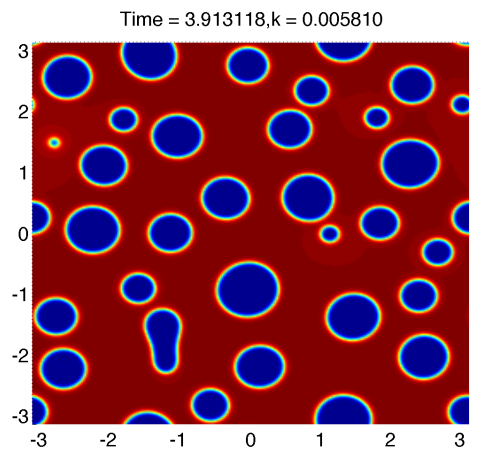

(c)

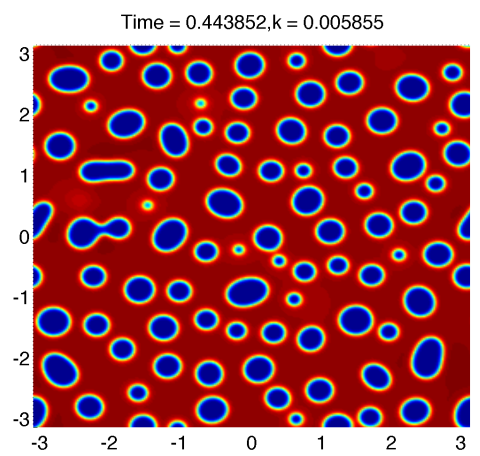

(b)

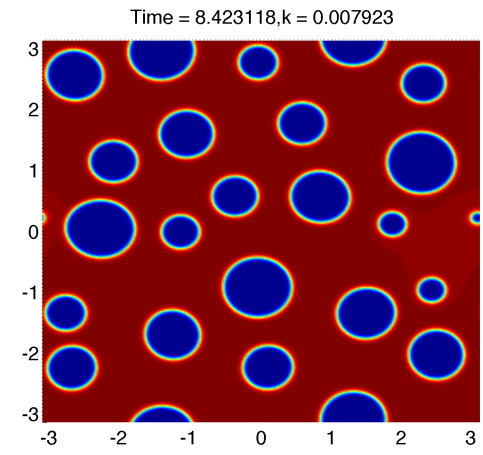

(d)

Fig. 3.1: Test 1: Phase separation and grain coarsening at early stage.

Test 1: Time steps. In this experiment, we choose $\tau=0.5$ at the early simulation stage and $\tau=100$ at the later stage. The constant $C$ in the iterative scheme (2.11) is chosen to be $3\left\|u^{n}\right\|_{\infty}^{2}$, which is slightly larger than 3 . The initial condition is set to be randomly created data such that $-1 \leq u \leq 1$ on the rectangular region $[-\pi, \pi] \times[-\pi, \pi]$. We impose periodic boundary conditions (other boundary 


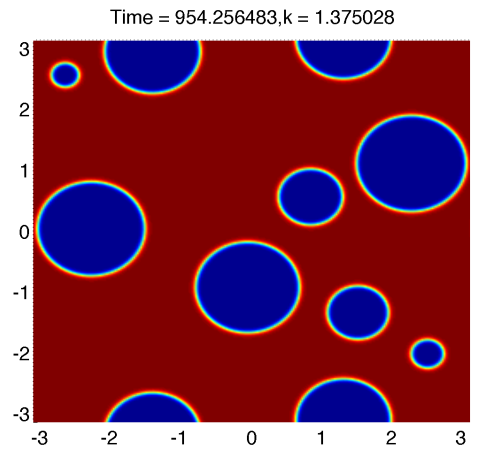

(a)

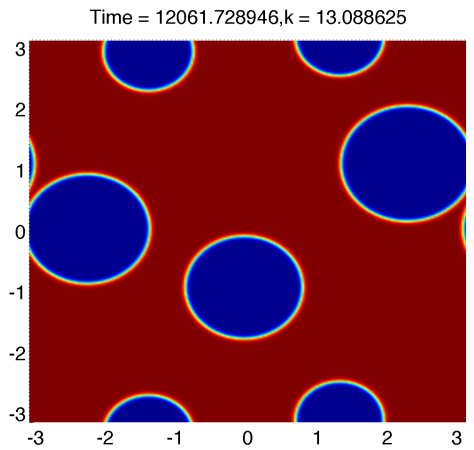

(c)

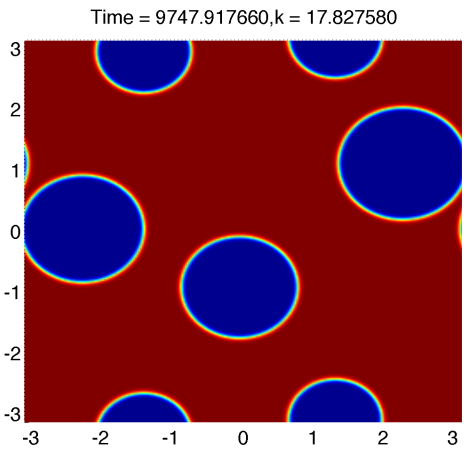

(b)

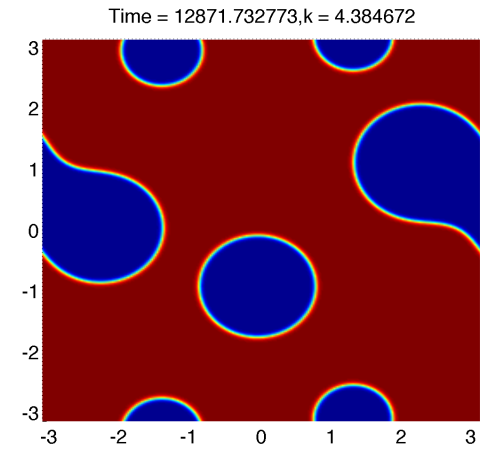

(d)

Fig. 3.2: Test 1: Late-time evolution with adapted large time steps.

conditions can be implemented in the pseudo-spectral method framework) to illustrate the effect of our approach.

In figure 3.1, we plot the contours of the solution for the early stage phase separation. We observe the active coarsening process which leads to radically changing small time steps due to the accuracy requirement in figure $3.3 \mathrm{a}$. Figure $3.3 \mathrm{~b}$ shows that the total energy decays with time. In figure 3.2 , which demonstrates the latetime evolution, we can observe the slow coarsening process. Figure $3.3 \mathrm{c}$ shows that a large adapted time step size ( $\mathbf{k}$ is about 100) is used to speed up the simulation. In figure $3.3 \mathrm{~d}$, it is shown numerically that the total energy decays at a very slow pace. Consistent with the underlying physics of the model, we observe that the mass of small grains is transported to large grains diffusively subject to the minimizing of surface area or the total energy. The main point here is that even when large time steps are used, the nonlinear iteration given by (2.11) still converges to the energy descent solution. The resolution of the interface structure can be compared with the result obtained by other high order methods, for example [19].

Test 2: Refinement study. In this numerical test, we would like to show that the solution given by the iteration method converges while the time steps are decreased. We start from initial condition with four shaped pieces and simulate the coarsening process to the time $T=1$. For $\tau=0.4$, we use the previously described adaptive time stepping strategy to obtain the initial time steps. During 


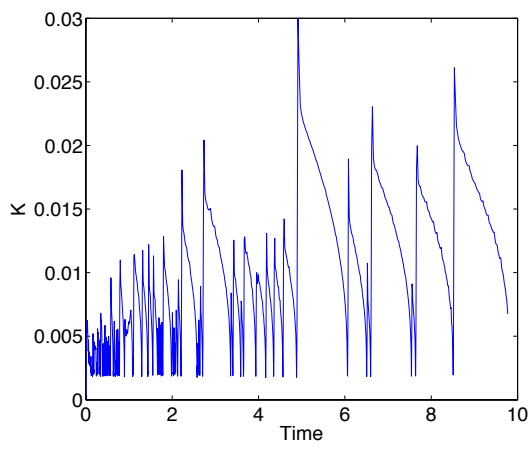

(a) Adapted time steps at early phase separation

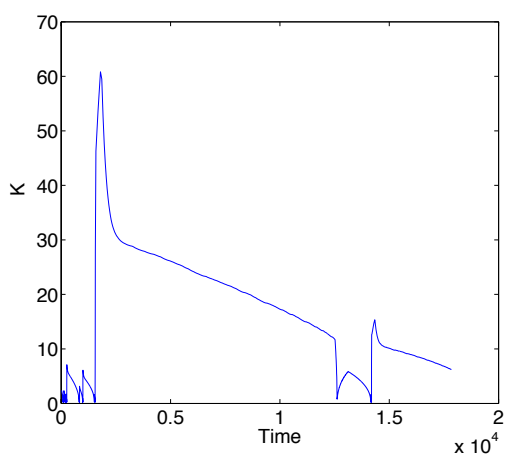

(c) Large adapted time steps at late time

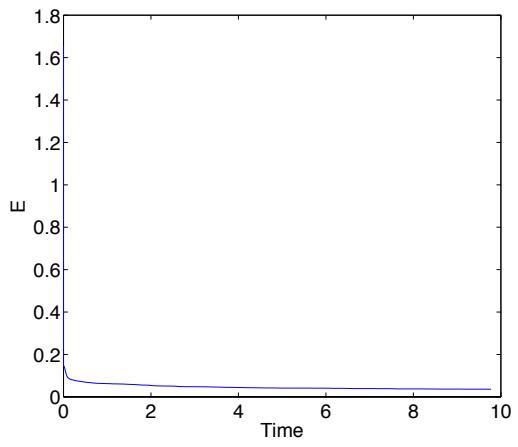

(b) Energy descent at early stage

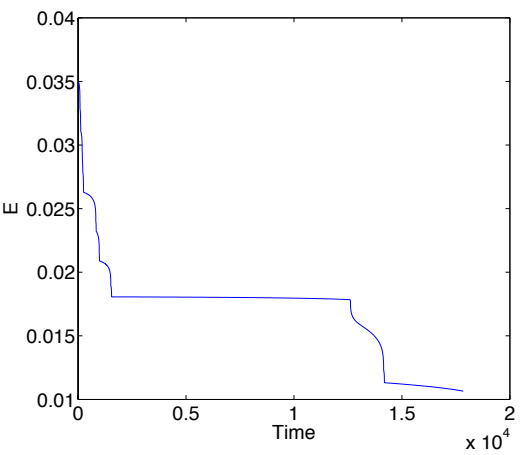

(d) Energy decent at late time

Fig. 3.3: Test 1: Energy descent with adapted time steps.

each refinement, we divide each time step by 2 to obtain refined time steps. In figure $3.4 \mathrm{c}$, we can observe that the solution converges while refining. The convergence rate in figure $3.4 \mathrm{~d}$ is close to first order, which is expected since the scheme is at most first order accurate in time discretization.

Test 3: Numerical error on the dynamics. In our first numerical test, we concluded that the time step constraint can be eliminated by the unconditionally gradient stable scheme. The iteration method itself also allows for large time steps. Therefore, the method gives us the flexibility to choose time steps based on the accuracy requirement. Here we cautiously extend our discussion to the impact of large time steps on the dynamics under simulation. Without doubt, in general, a less accurate time integration strategy and large time steps lead to a less accurate approximate solution to the PDEs under consideration. However, we would like to show through this numerical test that the numerical dynamics associated with a pre-defined energy form, produced by an energy descent scheme, are still accurate while large time steps are used. To illustrate this point, we start with a non-trivial initial data in figure 3.5a. We run the numerical simulation with three sets of time steps, $\tau=0.5,2,32$, which vary significantly.

For the three different time steps, we obtain similar schematic evolution of the 


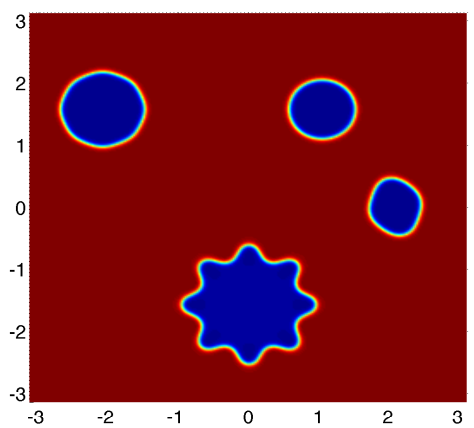

(a) Initial data

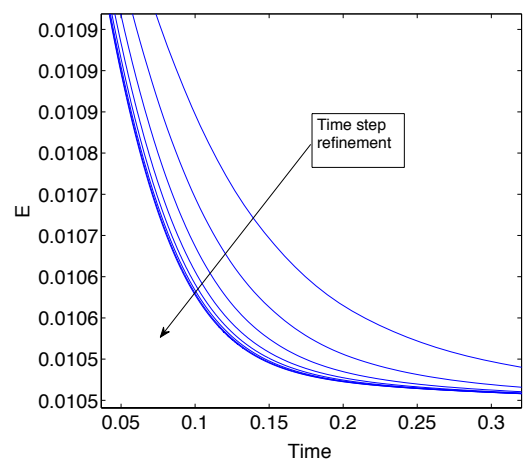

(c) Energy evolution for consecutively refined time steps

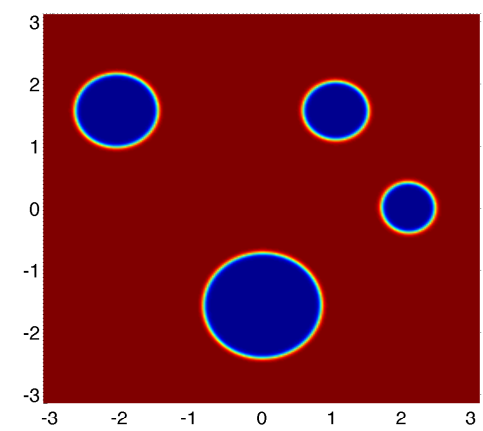

(b) End state at $\mathrm{T}=1$

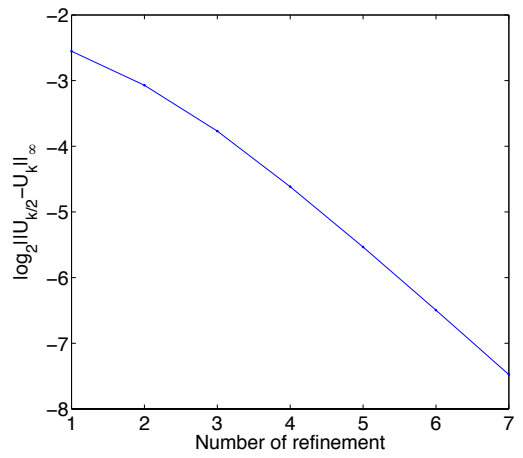

(d) $L^{\infty}$ error at $\mathrm{T}=1$

Fig. 3.4: Test 2: Refinement study and order of accuracy.

system, which is shown in the four contour plots in figure 3.5.

The curves in figure 3.6a show that the dynamics is stretched to a longer time to complete the ripening, due to numerical error caused by large time steps. Nonetheless, the evolution of the energy is very similar for these three different time steps. Moreover, figure 3.6b shows that taking large time steps requires fewer steps, and therefore less computational work to reach the minimal energy state. To verify that the computation is still accurate with large time steps, we plot the evolution of the mass center in figures 3.6c, 3.6d. We find that for widely different time steps, the final ripening states are very close, with a difference on the $10^{-3}$ level. The trajectory of the mass center is given in figure 3.7. We can observe that the trajectories do not digress far away from each other for widely varied time steps. In this sense, we deem that the scheme is still accurate for large time steps.

\section{Conclusion}

We reviewed some basic properties of a nonlinear semi-implicit scheme for phase field models. The scheme is proven to be unconditionally gradient stable, allowing large time-marching steps suited for efficient simulation of the long time evolution. In the framework of the Fourier method, we proposed a novel iteration method to compute the updated solution at each time step. We proved that the iteration itself 


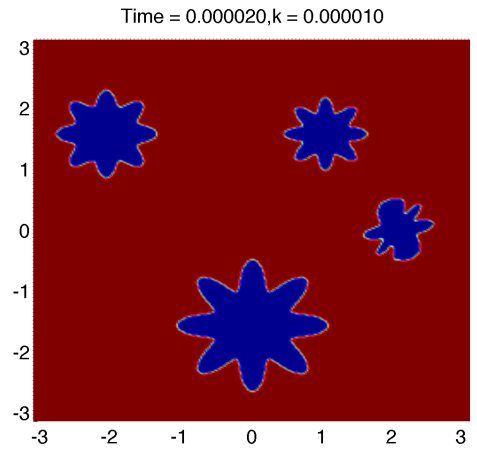

(a)

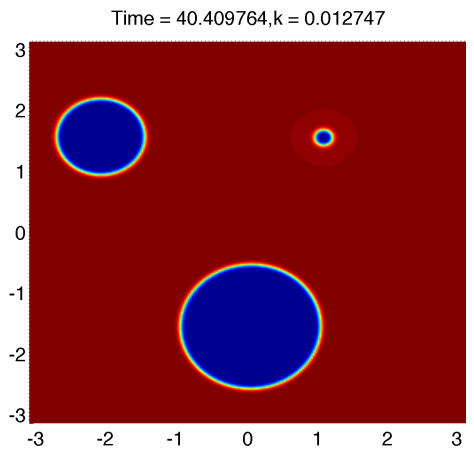

(c)

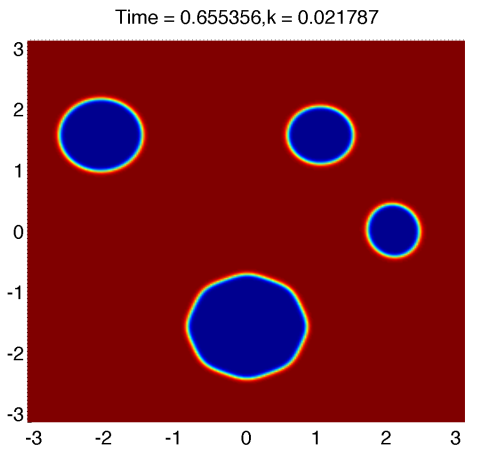

(b)

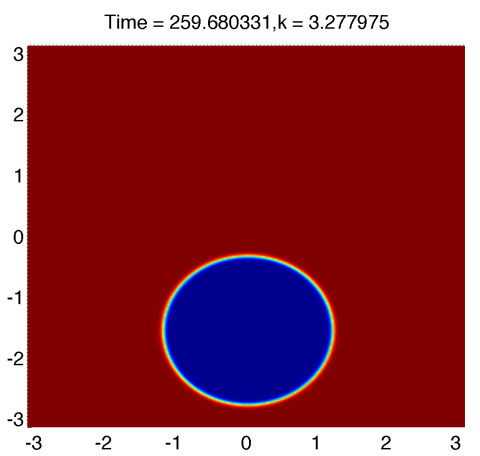

(d)

Fig. 3.5: Test 3: Schematic coarsening when $\tau=0.5$.

indeed is a contractive mapping for the Cahn-Hilliard equation under a time step size constraint. The proof relies on the estimate of the point-wise maximal value. For the Allen-Cahn equation, we showed that the proposed iterative method is a contractive mapping for large time steps, without obvious dependence on the layer width of the interface and the mesh size. Numerical results provided concrete evidence that the scheme works for simulation of the Cahn-Hilliard equation with large time steps. The technique is easy to implement and can be applied to general potential wells. Numerically, we discussed the impact of the numerical error on the dynamics associated with the pre-defined energy form. 


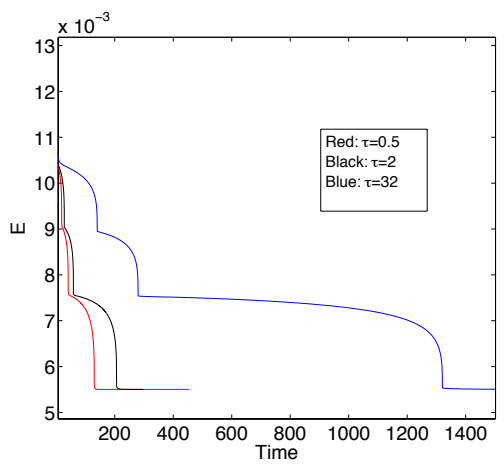

(a) Energy following simulation time

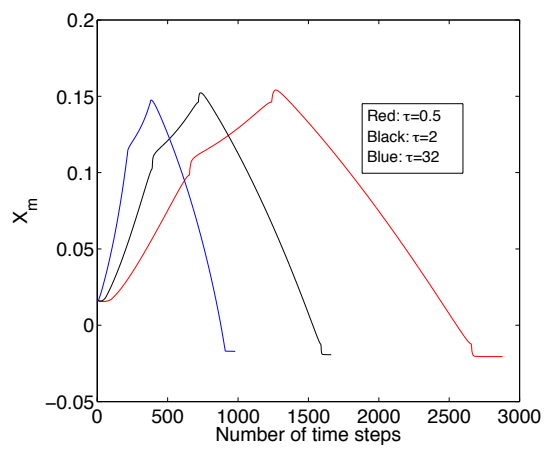

(c) X coordinate of mass center

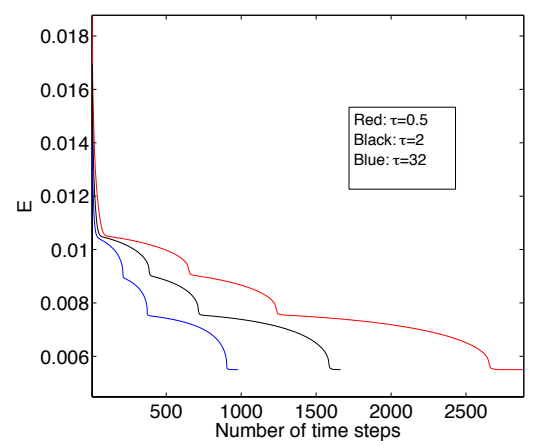

(b) Energy following number of time steps

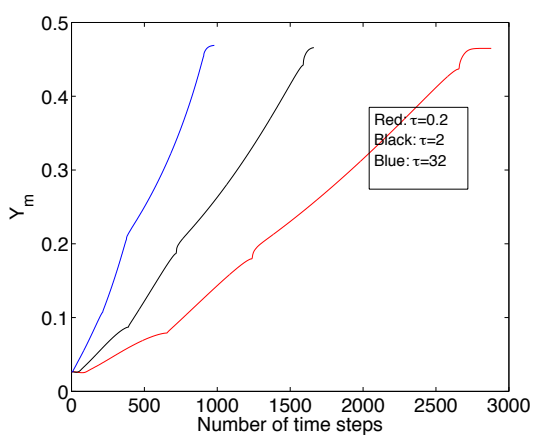

(d) Y coordinate of mass center

Fig. 3.6: Test 3: Energy for large time steps and history of mass centers.

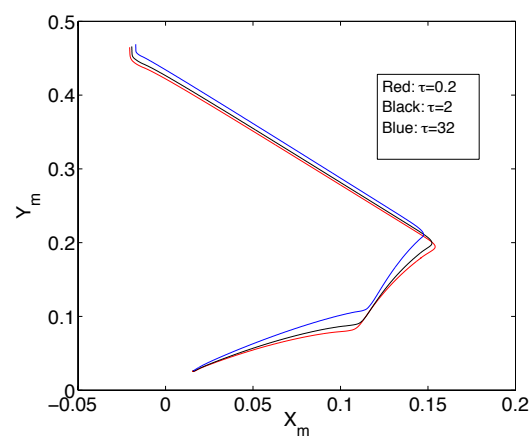

Fig. 3.7: Test 3: Evolution of mass center for different time steps.

Acknowledgment. The authors Zhengfu Xu and Andrew Christlieb are supported by NSF DMS 0934568 "Design and Development of efficient solid-state Dyesensitized solar cells." The author Keith Promislow would like to acknowledge the support of NSF DMS 0708804. 


\section{REFERENCES}

[1] A.J. Bray, Theory of phase-ordering kinetics, Adv. Phys., 43, 357, 1994.

[2] J.W. Barett, J.F. Blowey, and H. Garcke, Finite element approximation of the Cahn-Hilliard equation with degenerate mobility, SIAM J. Numer. Anal., 37, 286-318, 1999.

[3] J.W. Cahn and J.E. Hilliard, Free energy of non-uniform system. I. Interfacial free energy, J. Chem. Phys., 28, 258-267, 1958.

[4] L.Q. Chen, Phase-field models for microstructure evolution, Ann. Rev. Mat. Res., 32, 113, 2002.

[5] R. Choksi, M.A. Peletier, and J.F. Williams, On the phase diagram for microphase separation of diblock copolymers: An approach via a nonlocal Cahn-Hilliard functional, SIAM J. Appl. Math., 69, 1712-1738, 2009.

[6] Q. Du and R.A. Nicolaides, Numerical analysis of a continuum model of phase transition, SIAM J. Numer. Anal., 28, 1310-1322, 1991.

[7] C. Elliott and D. French, Numerical studies of the Cahn-Hilliard equation for phase separation, IMA J. Appl. Math., 38, 97-128, 1987.

[8] C. Elliott and Z. Songmu, On the Cahn-Hilliard equations, Arch. Rat. Mech. Anal., 96, 339-357, 1986.

[9] D.J. Eyre, An unconditionally stable one-step scheme for gradient systems, preprint.

[10] D.J. Eyre, Unconditionally gradient stable time marching the Cahn-Hilliard equation, Materials Research Society Symposium, 26, 1998.

[11] X. Feng and A. Prohl, Error analysis of a mixed finite element method for the Cahn-Hilliard equation, Numer. Math., 99, 47-84, 2004.

[12] D. Kay and R. Welford, A multigrid finite element solver for the Cahn-Hilliard equation, J. Comput. Phys., 212, 288-304, 2006.

[13] J. Kim, K. Kang, and J. Lowengrub, Conservative multigrid method for Cahn-Hilliard fluids, J. Comput. Phys., 193, 511-543, 2004.

[14] J. Lowengrub and L. Truskinovsky, Quasi-incompressible Cahn-Hilliard fluids and topological transitions, Proc. R. Soc. Lond. A, 454, 2617-2645, 1998.

[15] J. Zhu, L.Q. Chen, J. Shen, and V. Tikare, Coarsening kinetics from a variable-mobility CahnHilliard equation: Application of a semi-implicit Fourier spectral method, Phys. Rev. E 60, 4, 1999.

[16] J. Shen and X. Yang, Numerical approximations of Allen-Cahn and Cahn-Hilliard equations, Disc. Cont. Dyn. Sys., 28, 1669-1691, 2010.

[17] B.P. Vollmayr-Lee and A.D. Rutenberg, Fast and accurate coarsening simulation with an unconditionally stable time step, Phys. Rev. E 68, 066703, 2003.

[18] G.N. Wells, E. Kuhl, and K. Garikipati, A discontinuous Galerkin method for the Cahn-Hilliard equation, J. Comut. Phys., 218, 860-877, 2006.

[19] Y. Xia, Y. Xu, and C.-W. Shu, Local discontinuous Galerkin methods for the Cahn-Hilliard type equations, J. Comput. Phys., 227, 472-491, 2007.

[20] Z. Xu, A. Christlieb, and K. Promislow, Fast and accurate simulation of long time adiabatic evolution of polymer solvent system with gradient stable scheme, preprint.

[21] X. Ye, The Legendre collocation method for the Cahn-Hilliard equation, J. Comput. Appl. Math., 150, 87-108, 2003.

[22] P. Yue, C. Zhou, and J.J. Feng, Sharp interface limit of the Cahn-Hilliard model for moving contact lines, J. Fluid Mech., 645, 279-294, 2010. 